Numerical study on the generation of a planar supersonic gas-jet

\author{
M. Putignano, C.P.Welsch
}




\title{
Numerical study on the generation of a planar supersonic gas-jet
}

\author{
M. Putignano ${ }^{\mathrm{a}, \mathrm{b}, *}$, C.P. Welsch ${ }^{\mathrm{b}}$ \\ a The Cockcroft Institute, Daresbury Laboratory, Warrington, UK \\ ${ }^{\mathrm{b}}$ The University of Liverpool, Liverpool, UK
}

\section{A R T I C L E I N F O}

\section{Article history:}

Received 6 May 2011

Received in revised form

4 October 2011

Accepted 17 November 2011

Available online 26 November 2011

\section{Keywords:}

Gas-jet

Planar jet

Density profile optimization

Navier-Stokes

Compressible flow

Shock waves

\begin{abstract}
A B S T R A C T
Planar supersonic gas-jets are valuable tools for different applications such as state of the art molecular spectroscopy and accelerator beam instrumentation; however, the details of their expansion and generation have not yet been analyzed extensively, despite numerous studies addressing these same questions for the simpler case of the axis-symmetric jet.

In this paper, we investigate numerically the generation and evolution of planar supersonic gas-jets, extending the optimization studies done for axis-symmetric jets and focusing in particular on applications in accelerator beam instrumentation. We introduce a set of dedicated quality factors and use them to investigate the effects of changes to the geometry and thermodynamic state of the nozzle-skimmer system used to generate the planar jet. This analysis leads to an innovative, optimized nozzle-skimmer system design, which features advantages in terms of planar jet quality when compared to traditional setups. The proposed design also gives the possibility to modify, by variation of the thermodynamic quantities alone, the gas-jet characteristics in a wide range, including jet splitting and local density modulation. Density profiles of the generated jets are plotted for different parameter set.
\end{abstract}

(c) 2011 Published by Elsevier B.V.

\section{Introduction}

In the last decades, supersonic gas-jets created by letting a gas expand freely across a large pressure differential through a suitable orifice (nozzle), have found application in numerous fields of science and technology, ranging from reactive engines in aeronautics to laser machining [1,2]. Such applications have triggered a rich literature analyzing in detail the characteristics of the simplest form of gas-jet: the axis-symmetric gas-jet typical of aircrafts' exhaust plumes. Furthermore, due to their properties of low internal temperature and high directionality, gas-jets have attracted much interest as suitable targets for interactions in molecular spectroscopy [3], nuclear fusion [4] and atomic physics [5-7], for which momentum monochromaticity is a very important requisite of the target. In these applications, a pencil like gas beam is extracted from the expanding jet by means of conical collimators (skimmers), yielding a well localized neutral gas target.

There exists, though, a second family of applications in which the gas-jet needs instead to be shaped into a thin screen rather than a cylindrical beam; convenient application of planar jets to achieve higher sensitivity molecular spectroscopy was already reported in 1981 [8], whilst advantages justifying its application in accelerator beam instrumentation, where gas-jet directionality

\footnotetext{
* Corresponding author at: The Cockcroft Institute, Daresbury Laboratory, Keckwick Lane 1, Warrington, Cheshire WA4 4AD, UK. Tel.: +44 1925864056

E-mail address: massimilianoputignano@quasar-group.org (M. Putignano).
}

can be exploited for efficient pumping, have been shown in the latest years [9-11].

While many theoretical studies have been carried out on the axissymmetric gas-jet system and its interaction with the added conical skimmers [12-14], there have been no studies which expand these analysis to the bi-dimensional case of the planar jet collimated by means of slit skimmers, in spite of its wide applicability and the ready availability of slit skimmers, which also have several applications [15]. Rather, in the applications the gas screen is generally formed out of a large axis-symmetric jet either by collimation [9] or by focusing a molecular gas with a sizeable magnetic moment using a strong magnetic field [16-18]. An example of a typical gas-jet experimental apparatus, as used in atomic physics (axis-symmetric jet) and accelerator beam instrumentation (planar jet) is sketched in Fig. 1: except for the focusing magnet proposed by Fujisawa and co-workers [19], the two setups only differ in the geometry of the skimmers used.

Given the numerous applications of supersonic gas-jets, moreover, the requirements and figures of merit appropriate to the jet are also many and diverse. The most commonly used are jet density and temperature, which are relevant to many applications. In some applications in which the jet needs only to be used as a thin screen, however, like in the notable case of accelerator beam instrumentation, beam temperature is not of direct primary concern, being the focus more on the homogeneity of the created planar jet, and in the ability to efficiently evacuate it from the vacuum chamber. An alternative set of dedicated observables would thus be beneficial to the understanding of jet creation aimed at such applications. 


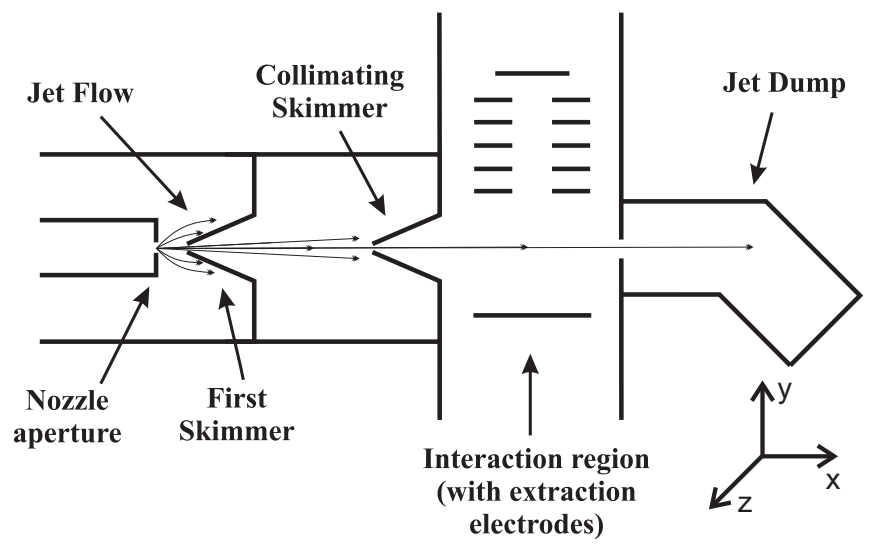

Fig. 1. Sketch of a typical experimental setup for the generation and use of the planar jet in atomic physics or accelerator beam instrumentation applications. The jet is created by expansion from the nozzle orifice and its innermost, supersonic core is extracted by means of the first skimmer, and further collimated with the second skimmer. It then travels through the interaction chamber, where the detector is located, and is finally dumped in a suitable pumping system.

To explore the physics of skimmer-collimated planar jets, and suggest alternative, better performing, dedicated means for its generation, we present in this paper a study of the influence on the jet structure of the nozzle-skimmer system and of the jet thermodynamic variables, putting the focus on applications in accelerator beam instrumentation. We furthermore introduce a set of quality factors for the assessment of the jet quality dedicated to applications requiring generation of a thin gas screen, and use them to suggest a novel, counterintuitive nozzle-skimmer geometry, characterized by a perpendicular, rather than parallel, orientation of the rectangular nozzle and skimmer. We show that this configuration outperforms the standard geometries otherwise used, and moreover allows tuning the density profile of the jet, obtaining for example a "split screen" profile, by only adjusting the temperature of the gas reservoir, without modifying any mechanical or geometrical parameter.

As far as gas species and density are concerned, in beam instrumentation, ideally a non-reactive monoatomic gas would be used to minimize vacuum pipe's' contamination, especially when superconducting sections are involved, and handling ease together with convenient ionization cross-sections suggest the use of Argon gas. Target gas density depends on the specific application, and in particular on the accelerated beam energy and intensity, and ranges from $10^{-7}$ particles $/ \mathrm{cm}^{3}$ for low energy, high intensity machines to $10^{-9}$ particles $/ \mathrm{cm}^{3}$ for high energy, low intensity accelerators.

\section{Numerical methods}

To fully describe the geometry of the nozzle-skimmer system and the thermodynamic variables of the flow, a large number of parameters are needed, resulting in the need to explore a vast multidimensional variable space. Therefore, a full experimental characterization of the system is unfeasible, and resorting to numerical analysis becomes compulsory.

\subsection{Simulation regime}

For the numerical simulations, we used a well established commercial code, the "Gas Dynamic Tool" (GDT), developed by the CFD group of A. Medvedev in Tula, Russia. The code has been widely benchmarked against known flows, proving very reliable in dealing with high compressibility effects such as shock waves [20].

Computationally, the most complicated case to study the planar jet expansion is the expansion at low pressures (sub-atmospheric), where transition to molecular flow occurs on a shorter scale; therefore, we focused our research on low pressure planar jets: any results obtained will thus be immediately extendable to higher pressure cases provided cluster formation stays negligible. Incidentally, moreover, low pressure jets are also the ones used in most applications of the planar jet, such as the mentioned cases of accelerator beam instrumentation. In these applications, the initial expansion stages of a typical gas-jet apparatus are housed in vacuum vessels which are kept at relatively high pressures of $10^{-2} / 10^{-4}$ mbar, while the jet itself has, in its expansion stage, a typical pressure of $1 / 0.1$ mbar. At room temperature, this leads to mean free paths in the sub-millimeter range, still compatible with the continuum description of the flow (Knudsen number $<0.2$ ). This allows the use of Navier-Stokes equations, which reduce to the Euler equations as it has been shown that the gas-jet expansion is a quasiisentropic process [12], and viscosity effects can be neglected. Hence, we have used the GDT continuum flow solver based on the Euler equations. If the mean free path needed in the particular application is longer than about a mm, the flow cannot be effectively described as a continuum flow anymore, and the description through the Navier-Stokes equations stops to provide reliable results: this sets the limits of applicability of our study. Throughout the paper, the gas investigated is Argon. Furthermore, we limited our analysis to the equilibrium condition of the gas-jet system, which is attained in a few milliseconds, depending on the particular system, its dimensions and the thermodynamic variables of the problem: therefore the analysis applies to continuous beams or pulsed beams with pulse duration significantly longer than the time needed to establish the equilibrium, provided the pumping speed of the system is sufficient to maintain the pressure conditions, as will be discussed in more details later.

\subsection{Variables and observables}

The analyzed system is a generalization of the nozzle-skimmer system for axis-symmetric jets: instead of using circular nozzle and skimmers, we let the nozzle and skimmer width/height ratio vary, obtaining the circular configuration as a particular case. The gas-jet expands from a high pressure reservoir through the slit nozzle into a region isothermal with the reservoir, and is collimated by the skimmer. This process selects gas atoms with quasi-parallel momenta, giving the flow its high directionality and monochromaticity. Murphy and co-workers showed that the structure of the jet is only affected by the nozzle internal geometry in the very first stages of expansion, i.e. a few nozzle diameters downstream the nozzle tip [21]. Our numerical simulations confirmed this result for the slit nozzle as well, which was therefore preferred to the more complex convergent-divergent configuration of the Laval nozzle.

This jet generation system was investigated varying 6 geometric variables, as shown in Fig. 2: the skimmer aperture angles ( $\alpha$ and $\beta$ ), the width of the skimmer slit $\left(S_{W}\right)$, the depth of the skimmer structure $\left(S_{D}\right)$, the nozzle-skimmer distance $\left(d_{n s}\right)$ and the width of the nozzle slit $\left(\mathrm{N}_{\mathrm{W}}\right)$. For consistency with the existing literature, all length units are normalized to the nozzle slit height, equivalent to the nozzle diameter for standard axis-symmetric setups, and are therefore dimensionless. The skimmer slit height is also kept equal to 1 . In addition to these parameters, we varied the pressure ratio between the gas reservoir and the expansion chamber $(R)$ and the common gas temperature in the two environments across the nozzle $(T)$.

Concerning the observables to monitor, a general formulation of the jet performance, suitable for most applications, can be done on the basis of three quality factors: jet geometry, density homogeneity and confinement. The quality of the geometry can be assessed by the ratio $G_{R}$ between the long and the short dimensions of the jet screen, defined as the FWHM of the density profile in the corresponding directions. The region enclosed by these dimensions will henceforth 


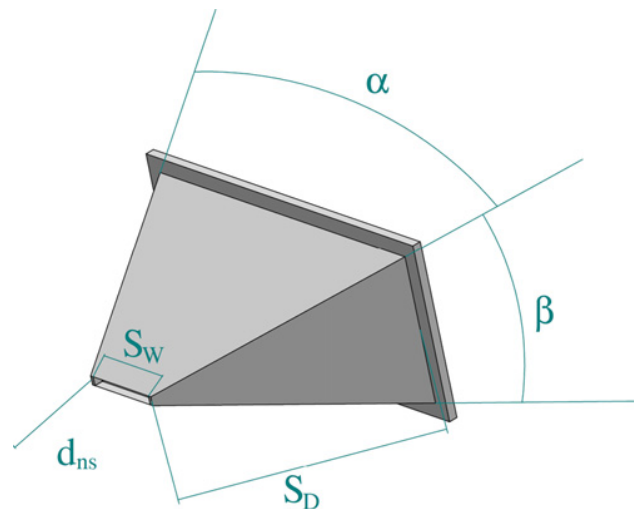

Fig. 2. Definition of the geometric variables of the skimmer.

be referred to as the screen. The density homogeneity $H_{\rho}$ is best expressed by the standard deviation of the density profile across the screen region, normalized to the mean density. The confinement $K$ relates to the sharpness with which the tails of the density profile roll off, and is expressed by the percentage of total gas mass past the skimmer enclosed in the screen region. Because of their definitions all three quality parameters are dimensionless.

These quality factors are computed downstream the skimmer at the coordinate when the hard sphere binary collision frequency drops to the point that the computed Knudsen number exceeds 0.2 , the continuum description of the flow fails and the system approaches the molecular flow region where inter-gas collisions rarefy until their effect becomes negligible within the scale of up to few meters typical of the jet application. Since only a negligible number of collisions occur past this point, the gas properties are frozen to their terminal value [22]. From this point on, the cross section of the jet only scales geometrically due to the spread in momenta, making this the first point in the jet expansion where it is possible to perform a comparison between different nozzleskimmer systems jet performances.

\section{Boundary conditions}

To obtain precise data, the boundary conditions settings are of crucial importance. When the system of gas-dynamic equations in the non-stationary formulation is solved, to analyze an equilibrium condition it is necessary to simulate it from the beginning to the attainment of the steady state. Boundary conditions will prove influent in such a process insofar as the domain dimensions are small enough that a perturbation can travel from the region of interest to the boundary and back before the steady state is reached. This will normally be the case, as otherwise a too large domain would have to be used, critically increasing computation time. In turn, the same need for a small enough domain results in the need to introduce artificial boundaries in the flow in regions, which have no physical significance like a solid object or another fluid, but are simply the point where we want our simulation domain to stop, assuming no other significant event is happening outside this domain. The introduction of these artificial boundaries invariably creates artifact distortions in the simulated flow, which are well documented in literature [23]. In particular, these artifacts rise from the spurious reflections of waves from inside the domain on the domain boundaries, if these are not transparent. Several attempts have been made in the literature to achieve a mathematical formulation of transparent boundaries, which minimize the artifact distortions in the simulated flow, and these modified boundaries conditions are known as open boundary conditions, or Non-Reflective Boundary Conditions (NRBC). From what has been said, it is clear that when there is a large amount of gas flowing at supersonic speed through a border, NRBC boundary conditions are essential as they are transparent to the perturbation, which is lost beyond the boundary, whilst fixed value boundary conditions would instead result in the occurrence of artifact reflected shock waves. The NRBC used by the GDT solver are based on the Sommerfeld radiation conditions [25], which is described by Eq. (1):

$\frac{\partial u}{\partial t}+C u_{n}=0$

where $u$ is the generic variable, $C$ the waves phase velocity and $u_{n}$ is normal to the boundary.

When there is no considerable gas flow through a boundary, fixed value boundary conditions are preferable over NRBC both for reduced computational weight and results stability; if NRBC are used in such situation, local oscillations of pressure and density values are amplified, resulting in unstable motion.

To show the importance of these numerical effects, we simulated the well known bi-dimensional solution of free supersonic expansion by a capillary orifice, and measured the dimensions that describe the shock wave pattern (Mach disk diameter and distance from nozzle, barrel shock diameter, triple point diameter) [22]. For these simulations the pressure in the gas reservoir is set at 1 bar and the vacuum chamber has an initial base pressure of 0.1 mbar. In the case of our simulations, the domain was chosen to be a rectangle for $2 \mathrm{D}$ simulations and a rectangular box for 3D simulations. GDT allows setting different boundary conditions on each of the sides of the rectangle/faces of the box, or even in different regions of the same face. In the 2D case, given the need to use fixed value boundary conditions on the left side of the rectangle, since that represents the high pressure reservoir, 3 sides are left to choose between fixed value and NRBC. If all three boundaries are set as open, it is observed that the gas-jet expands as predicted up to the expected equilibrium condition. This steady state stays stable only over a very short time of less than $100 \mu \mathrm{s}$. Soon after, it starts wobbling and becomes unstable until, only about $0.5 \mathrm{~ms}$ after the start of the process (depending on the thermodynamic parameters and on the dimensions of the computation domain), it grows out of the steady state and starts expanding up to the filling of the whole domain. Such time instability is shown in Fig. 3, where subsequent stages of the process are illustrated.

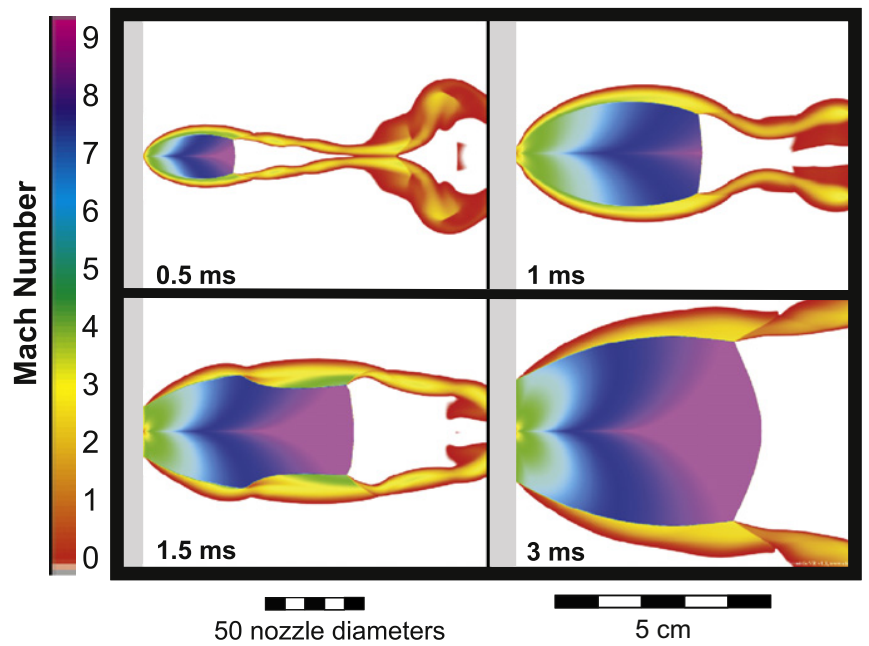

Fig. 3. Subsequent stages (top line $0.5 \mathrm{~ms} ; 1 \mathrm{~ms}$; bottom line $1.5 \mathrm{~ms} ; 3 \mathrm{~ms}$ ) of the free expansion of an axis-symmetric gas-jet computed using NRBC conditions on all three gas boundaries. Time instability of the equilibrium pattern is shown. The flow reaches a first condition of equilibrium (top left); then expands further and reaches a second position of equilibrium (top right); only to show some instabilities (bottom left) and finally blowing up (bottom right) until it eventually exceeds the domain. The pressure in the high pressure reservoir is considered to be at full value already before the simulation begins, hence modeling a step rise. 


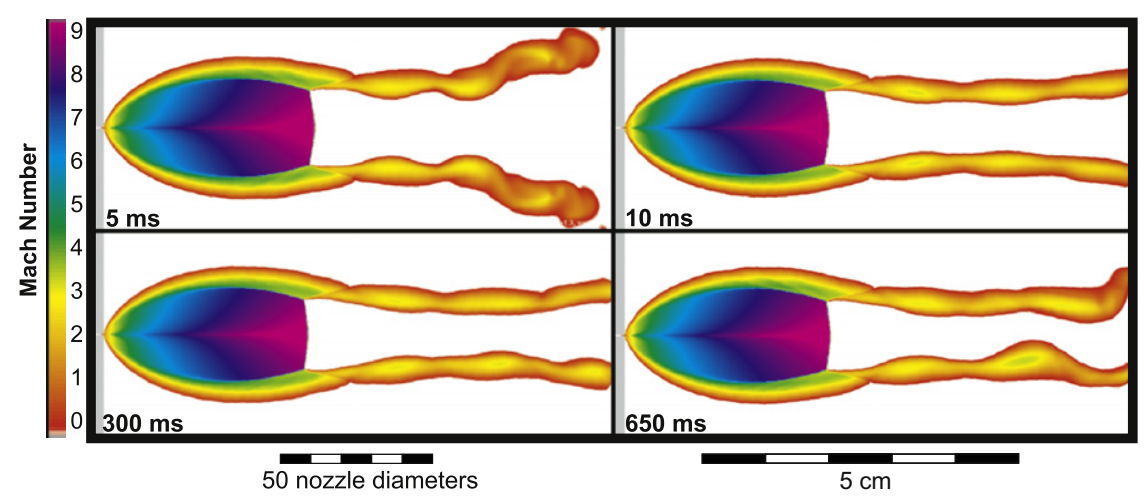

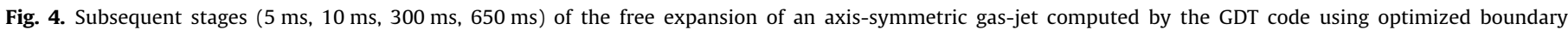

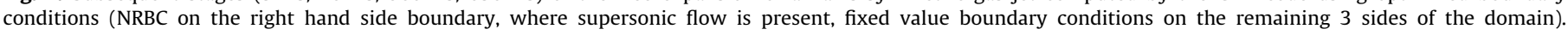

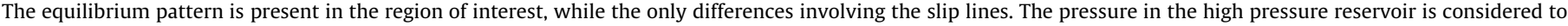
be at full value already before the simulation begins, hence modeling a step rise.

Much better results are obtained by setting the boundary regions not exposed to supersonic flow to constant fixed pressure, in order to stabilize the results. In the regions where supersonic flow is not negligible, mainly downstream the expansion, NRBC are preferred, to avoid reflection of artifact shock waves formed at the interface between the flow and the constant pressure boundary.

When this is done, the reflected shock waves at the right boundary are found to interfere mainly with the slip lines, modifying their shape and thickness, but featuring only a negligible influence on the region of interest. In fact, this improvement is so pronounced that it was possible to halve the vertical dimension of the simulation domain, bringing the boundary much closer to the region of interest where shock waves are present. This is a proof of the efficiency of the boundary conditions chosen, as proximity of the boundary to the region of interest without creating large artefact waves is one of the main figures of merit when assessing boundary conditions performance. Quantitatively, the improvement can be seen from the comparison of the shock waves structure, up to the Mach disk, in the 4 different cases, corresponding to different times of the expansion, shown in Fig. 4. In particular, we monitored the Mach disk position, the Mach disk diameter and the barrel shock diameter, and found them to be constant within $<7.5 \%$ in all the simulated time $(650 \mathrm{~ms})$ after attainment of the equilibrium state (about $100 \mu \mathrm{s}$ after start of expansion). This indicates that the system does not change after the time needed for the perturbations to travel to the boundary and back, and that, thus, these artifact reflections are only creating an effect below $7.5 \%$ on the measured shock wave structure.

The GDT code was furthermore expanded with purposewritten $\mathrm{C}++$ analysis modules which automate variables modification and simulation runs, import data from GDT and compute and display observables of interest, greatly enhancing the ability of GDT to run and compare large numbers of simulations.

\section{Results: configuration performance comparison}

Because of the large number of variables involved, computation time becomes an issue. To minimize it, we run a set of 100 exploratory simulations with different variables combinations, aiming at establishing the time needed for the system to reach equilibrium. The tests showed that in all conditions tested the density values in the simulation domain fall within $5 \%$ of the long term equilibrium solution (simulated up to $10 \mathrm{~ms}$ ) within $750 \mu \mathrm{s}$, which was thus taken as our simulation runtime, bringing the computation time to about $550 \mathrm{~s}$ per simulation on a Quad-Core Intel ${ }^{\mathbb{R}}$ Xeon $^{\mathbb{R}}$ 3.40 GHz Processor. The simulation grid used included 5M points.
We run about 8000 simulations, exploring the whole variable space by assigning 3 different values to each variable, in both the perpendicular and parallel nozzle-skimmer slits configuration.

A subset of these simulations, showing only 2 different values for each variable, is shown in Fig. 5. On the x-axis is the identification number of each simulation, whose variable set can be read on the $y$ axis in Fig. 5a. On the y-axis of Fig. 5b-d are plotted the resulting density homogeneity $H_{\rho}$, geometrical ratio $G_{R}$ and confinement $K$.

Four combinations turn out to be of particular interest as they resemble commonly used configurations: (a) circular nozzle and skimmer; (b) circular nozzle and slit skimmer; (c) slit nozzle and slit skimmer parallel and (d) perpendicular to each other, corresponding, respectively, (see Fig. 5a) to simulations number 1-64; 65-128; 129-192 and 193-256. It must be noted that in configuration (a), since the nozzle has circular symmetry, angles $\alpha$ and $\beta$ are interchangeable, and therefore the simulations with small $\alpha$ and large $\beta$ have identical results to the ones with small $\beta$ and large $\alpha$ (the screen long and short dimensions are redefined by the data analysis code depending on the actual orientation of the screen). Furthermore, the geometric ratio for simulations in which $\alpha=\beta$ is always evaluated equal to 1 as the screen formed in this case is symmetric, and in practical applications a collimating slit is needed to form the planar jet.

In what follows we evaluate these configurations against each other on the basis of the numerical results, using as quality factors the values of $H_{\rho}^{-1}, G_{R}$, and $K$ for the configuration under exam, normalized to the values of the same observables obtained for configuration $\mathrm{d}$ : slit nozzle and skimmer perpendicular to each other. $H_{\rho}^{-1}$ is preferred to $H_{\rho}$ so that higher values mean better performance.

In order to give an indication of the overall influence of the configuration change alone, each normalized quality factor has been calculated for each competing configuration, a, b and c, and for all available combinations of other variables, and all these values averaged. For the purpose of optimization, it is instead more interesting to compare the best achievable value for each configuration.

Table 1 lists both the average values and the best values of $H_{\rho}^{-1}, G_{R}$, and $K$ for different configurations.

Table 1 shows that configuration d performs sensibly better than the others with the exception of the confinement, which is about $50 \%$ better in configuration $\mathrm{c}$.

In particular it can be seen how, using perpendicular slits for nozzle and skimmer, the homogeneity and the confinement of the planar jet are increased when compared with commonly used configurations with circular nozzle and also outperforms configuration c by a similar factor in homogeneity and geometric 
a

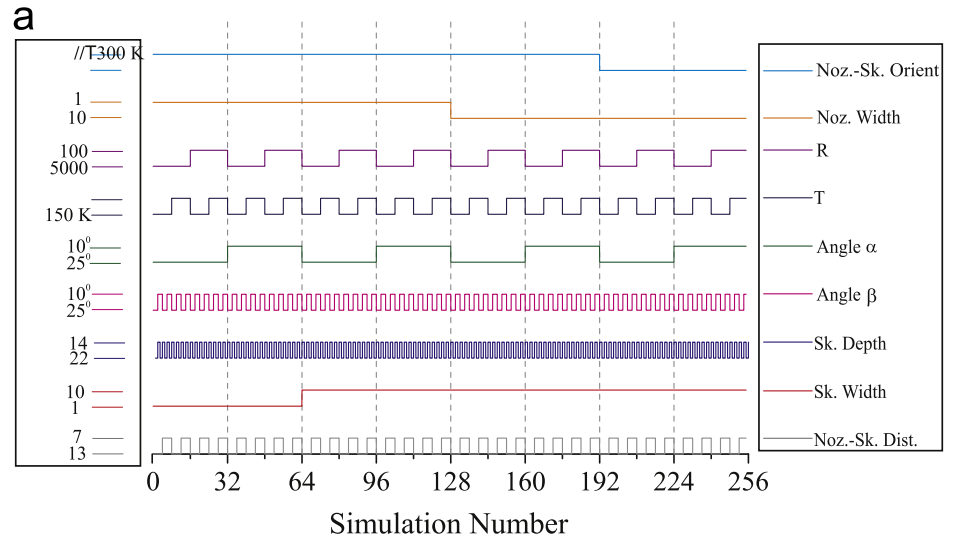

C

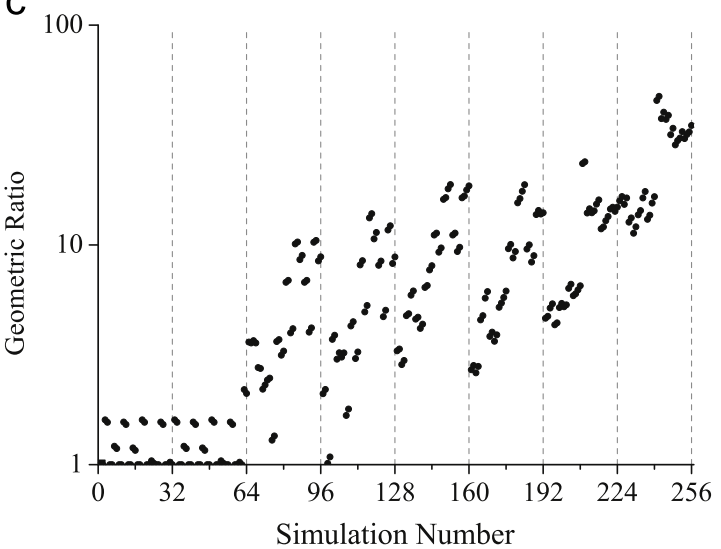

b

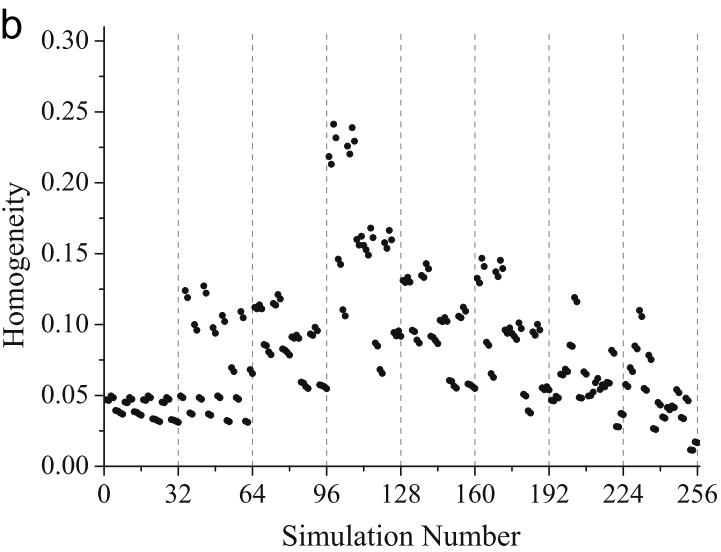

d

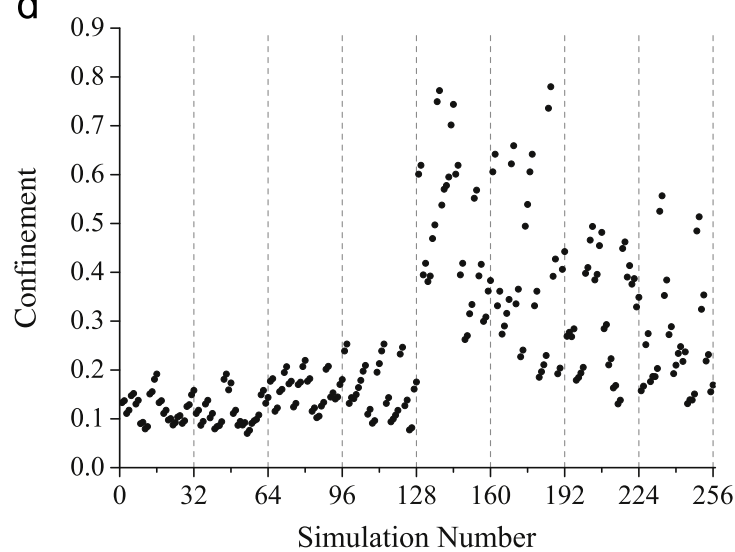

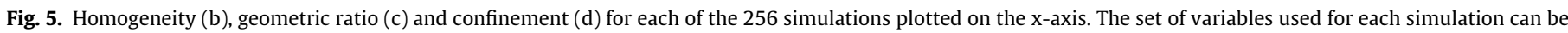
read on the $y$ axis of plot (a).

Table 1

Performance ratio between configuration d (slit nozzle and skimmer perpendicular to each other) and the competing configurations: a (circular nozzle and skimmer), b (circular nozzle and slit skimmer) and c (slit nozzle and skimmer parallel to each other). Ratios lower than 1 indicate a better performance of configuration $\mathrm{d}$.

\begin{tabular}{llllllll}
\hline & \multicolumn{1}{l}{ Average } & & & & \multicolumn{2}{l}{ Best value } \\
\cline { 2 - 3 } \cline { 6 - 8 } & $\mathrm{a}$ & $\mathrm{b}$ & $\mathrm{c}$ & & $\mathrm{a}$ & $\mathrm{b}$ & $\mathrm{c}$ \\
\hline$H_{\rho}^{-1}$ & 0.8 & 0.46 & 0.58 & & 0.37 & 0.21 & 0.30 \\
$G_{R}$ & 0.13 & 0.31 & 0.51 & & 0.09 & 0.29 & 0.40 \\
$K$ & 0.44 & 0.55 & 1.55 & & 0.48 & 0.45 & 1.40 \\
\hline
\end{tabular}

ratio, at the expenses of losing about $50 \%$ in confinement. The geometric ratio also increases when using configuration $\mathrm{d}$, in particular when compared to configuration a, in which of course one needs to use additional collimating slits to obtain a planar jet.

For practical applications, optimized performance is a more interesting parameter. It can be seen from the right hand side of Table 1 that the improvement in homogeneity and geometric ratio is sensibly increased. The same is true to a lesser extent for the confinement.

The factor of 2 to 3 that can be gained in geometric ratio using the proposed configuration d results directly in a correspondingly higher density available for the jet in its core (as the gas flowing outside the required geometric ratio does not need to be scraped out). This value can therefore be compared with the effect obtained by Hashimoto and co-workers [18] by the use of magnetic focusing, which was reported to be about 2 . It is thus an advantage of the configuration proposed that comparable performance improvement can be obtained without the further complication of an added magnet and corresponding field, beside not being restricted in the choice of gas to a molecular gas with a sizeable magnetic moment.

Finally, we mention that in practical applications, a further collimating slit or skimmer is used downstream the first skimmer. Given the nature of the flow, which at this point is no more thermodynamic, but molecular, this second skimmer collimates the jet simply geometrically; therefore by increasing the distance from the jet source to the target and reducing the aperture in the second skimmer, is possible to increase the geometric ratio indefinitely. However, this comes at the cost of reduced jet density at the target region. We choose not to include a collimating skimmer in our simulations, as it can be applied to any configuration and used to obtain identical effects: the performance increase in the configuration we propose decreases the need for additional collimation, allowing to use a larger portion of the jet in the target region and hence obtaining higher densities for the same values of stagnation pressure and temperature.

It should be also stressed again that the performance considerations discussed are based on the particular choice of observables, which is particularly suited to thin screen applications like beam instrumentation, and that optimized performance configuration can thus vary dramatically depending on the particular application.

\section{Results: variable by variable analysis}

Having identified a nozzle-skimmer configuration yielding best performances, it is possible to narrow down the variable space and run additional simulations with finer details to assess 
the impact of different geometric variables on the creation of the planar jet.

Fig. 6 shows two examples of this study: the first three plots (Fig. 6a-c) show how $H_{\rho}, G_{R}$ and $K$ change for increasing angle $\alpha$ (x-axis) and angle $\beta$ (curve parameter). The last three plots (Fig. 6a-c) show how $H_{\rho}, G_{R}$ and $K$ change for increasing skimmer slit width $S_{W}$ (x-axis), and angle $\alpha$ (curve parameter).

It is apparent from the first plot that the homogeneity of the screen slightly worsen when changing $\alpha$ from $5^{\circ}$ to about $15^{\circ}$ as the system moves away from a quasi-axis-symmetric configuration and more marked expansion fans are created past the skimmer, only to improve past $15^{\circ}$ due to a dramatic increase in geometric ratio which makes the frontier regions of the screen less influent on the overall homogeneity. Conversely, increasing $\beta$ only takes the system away from the quasi-axis-symmetric configuration, without the compensating effect of improving $G_{R}$. Therefore, increasing $\beta$ consistently leads to homogeneity worsening. Fig. 6b shows the behavior of $G_{R}$ when $\alpha$ and $\beta$ are increased, confirming the intuitive trend of $G_{R}$ improving with increasing $\alpha$ and decreasing $\beta$. Finally, analyzing Fig. $6 c$, it can be seen how extreme values of $\alpha$ lead to better confinement, with $K$ touching a minimum in the $10^{\circ}-20^{\circ}$ region, depending on $\beta$. This can be understood in terms of the density profile fall-off, which is sharper for small $\alpha$, due to the gas flow being restricted by the inner walls of the skimmer aperture, as it is also proved by the increasing influence of the skimmer depth for smaller and smaller aperture angles $\alpha$ and $\beta$ (see Fig. 6d-f); while conversely confinement improvement is again an effect of having large $G_{R}$ for large $\alpha$, leading to the creation of a larger screen, hence including more gas mass.

The behavior of $\alpha$ identified for all three observables $H_{\rho}, G_{R}$ and $K$ stays consistent also when $S_{W}$ is changed, as it can be seen in Fig. $6 \mathrm{~d}-\mathrm{f}$, where $\alpha$ is used as curve parameter. Furthermore, Fig. 6d shows that homogeneity is optimized for skimmer width matched with about half the Mach disk diameter, due to a trade off between efficient extraction of the coldest, innermost part of the jet, and $G_{R}$ increasing with larger $S_{W}$, which, as discussed above, makes the frontier regions of the screen less influent on the calculation of homogeneity. Indeed, with reference to Fig. 6e, $G_{R}$ improves with increasing skimmer width, as more gas is extracted from the supersonic expansion. This effect decreases as the skimmer width approaches the dimensions of the Mach disk shock wave, and the barrel shock boundary, which defines the boundary of the supersonic flow, is approached, preventing more gas to be extracted from the jet core. A confirmation of this was sought by running the simulations in Fig. 6e for increasing values of $R$, producing a larger jet, with larger Mach disk: in this case the improvement in $G_{R}$ is linear with $R$ as expected. Finally, confinement increases almost linearly with skimmer width, due to the skimmer width approaching the dimensions of the barrel shock, where the internal temperature of the jet has an abrupt drop, reflecting in a sharper fall-off of the density profile on which the confinement is calculated. Again, this effect shifts towards larger skimmer dimensions if $R$ is increased, furthering the barrel shock boundary and confirming the explanation above.

Given the amount of different variables, the results from a larger set of such plots are best summarized by identifying behavioral trends spotted when changing each variable and representing them schematically in a table.

A trend is intended to be found when the form of the functional relationship between the observable and the variable under investigation is preserved in the simulations regardless of the actual values of the other variables. This way, we are able to draw a table, shown in Fig. 7, which summarizes the simulated behavior of each observable (column entry) when the respective variable is increased (row entry). We identify linear relationships (straight arrows), parabolic relationships (curved arrows) and more complex relationships (circles), where even the form of the functional relationship depends on the value of some secondary variables (indicated inside the circle), and hence, according to our previous definition, a trend is not found. The latter is a qualitatively different behavior as compared to the first two cases, where the shape of the trend does not depend on the remaining variables, while still the details of the trend, such as the gradient for the linear relationships, will depend on the values of the remaining variables. In the table the bold lines represent very clear trends, defined as those trends where the
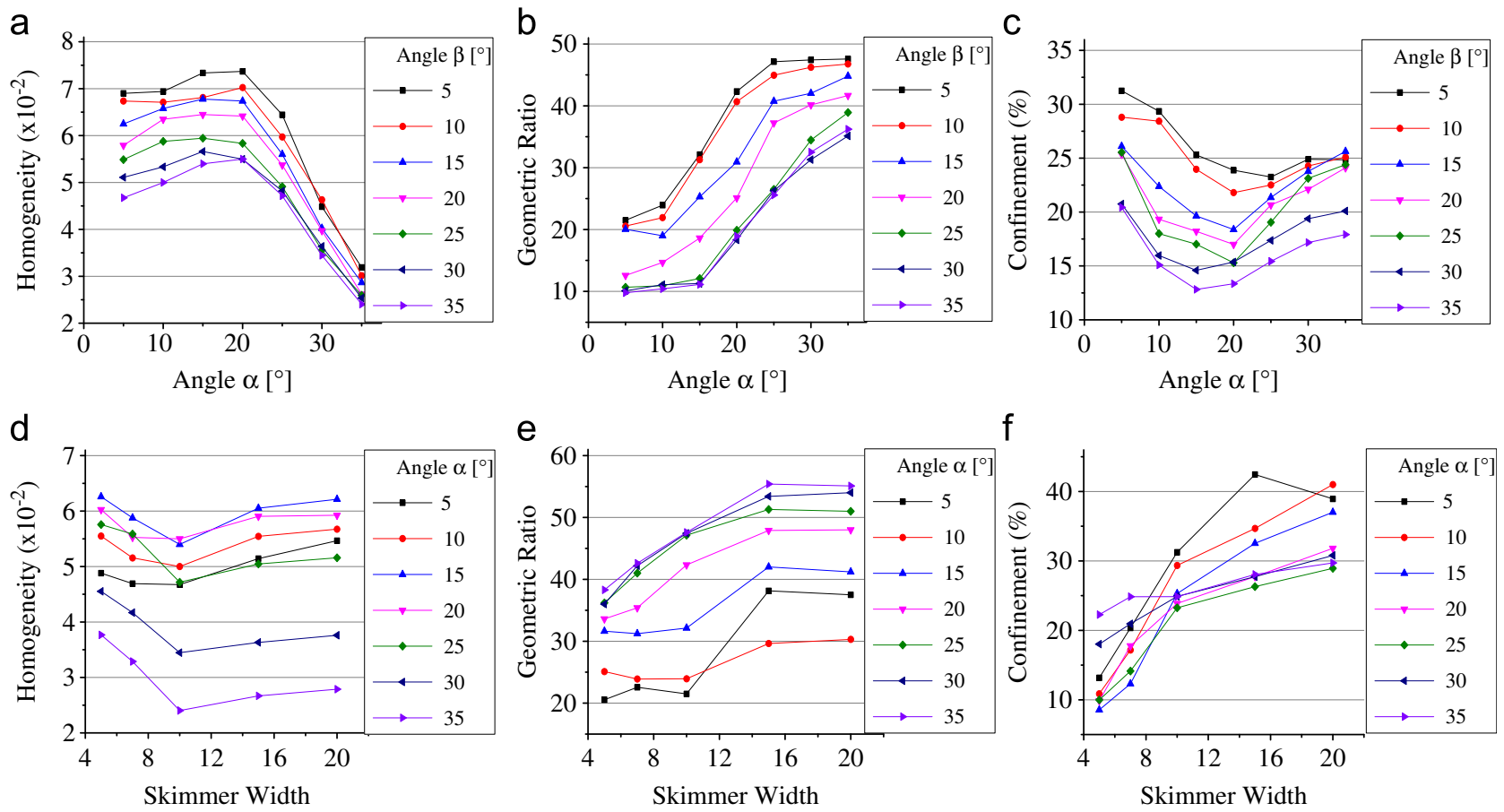

Fig. 6. Analysis of homogeneity, geometric ratio and confinement for finer variations of angles $\alpha$ and $\beta$ (a-c) and skimmer width (d-f). 


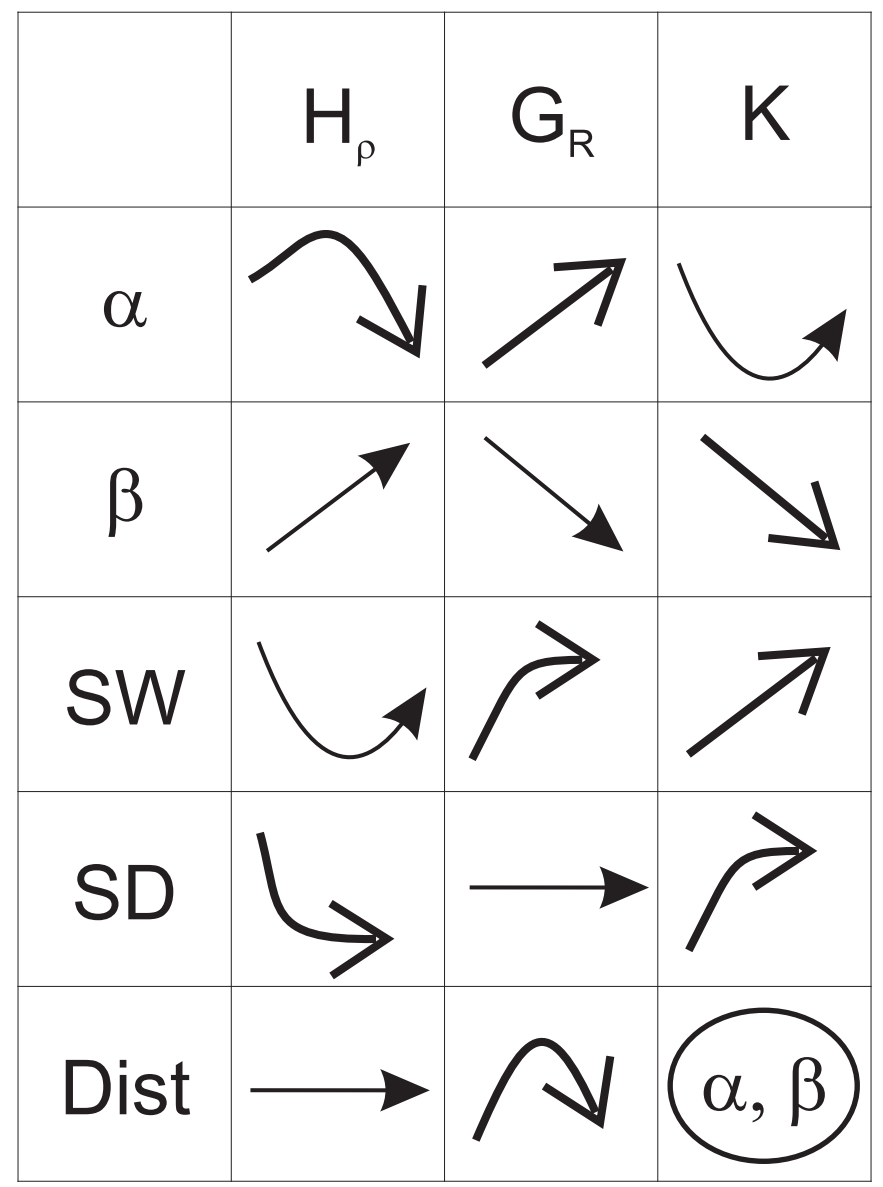

Fig. 7. Table of simulated trends, the arrows schematize the behavior of the column observable when the row variable is increased.

average overall points of the best fit Pearson value lies above $90 \%$, while the slimmer lines represents less evident trends, where the average best fit Pearson value lies between $75 \%$ and $90 \%$.

This table gives an indication of how sensitive the gas-jet is to the geometry of the nozzle-skimmer system. Furthermore, it also gives an insight as to which variables have a stronger impact on the performance of the jet in terms of homogeneity (namely $\alpha$ and $S_{D}$ ), geometric ratio $\left(\alpha, S_{W}\right.$ and $\left.d_{n s}\right)$ and confinement $\left(\beta, S_{W}\right.$ and $\left.S_{D}\right)$, besides giving guidance for directing experimental optimization efforts.

The data shown up to this point allowed us to identify an optimized configuration, which addresses several trade-offs between the three observables used and the technological limits. The following optimization discussion applies in particular to applications like e.g. accelerator beam instrumentation, for which $G_{R}$ and $H_{\rho}$ are the most important variables.

The crucial factor for jet performance is the pressure ratio $R$. Indeed, increasing $R$ always results in better performance for all observables; in particular, homogeneity decreases with the logarithm of $R$, and similar trends can be observed for geometric ratio and confinement, even if with less clear trends (Pearson value $<70 \%$ ). However, improvement with increasing $R$ is limited by cluster creations [27] and vacuum pumping speed available: in practice, ratios larger than $10^{6}$ are difficult to achieve. It must be noted that simulation parameters are set so that gas transport is possible across the simulation domain in presence of a pressure differential, with the pressure at the domain boundary set as the pressure limited by the given pumping available. Therefore the simulations yield realistic results at room temperature for any averagely large vacuum chamber ( $>20 \mathrm{~cm}$ ) if a pulsed jet is used with gate time $\leq 1 \mathrm{~ms}$, and this requirement relaxes linearly as the temperature decreases. Beyond this gate time, the pumping speed coming from the vacuum pumps and chamber geometry has to be taken into account, but the overall effect is that of a reduction of effective pressure ratio and hence results in decreased performance in all observables as discussed above.

The aperture angles of the skimmer also have a very important role. In general, increasing the angle $\alpha$ improves the performance of the jet with respect to $H_{\rho}$ and $G_{R}$, sacrificing only slightly the confinement with respect to configurations with small $\alpha$. However, too large values for $\alpha$ introduce severe distortions in the jet profile which can result at higher temperatures in jet splitting (see next section for details). We run further simulations at a significantly lower temperature, to confirm this trend, choosing for this purpose the temperature of $77 \mathrm{~K}$, in virtue of its ready availability through liquid nitrogen cooling. These simulations showed that indeed jet splitting does not occur at this temperature even for small $\left(<10^{\circ}\right.$ ) apertures angles. However, for stable operation at higher temperatures, $\alpha$ should be chosen between $25^{\circ}$ and $30^{\circ}$. Conversely, $\beta$ should be kept to about $5^{\circ}$ : while decreasing $\beta$ also proves beneficial to all observables, below $5^{\circ}$ the density profile becomes unstable, resulting in jet splitting at higher temperatures.

From what has been said, it is clear that temperature is an important parameter insofar as it allows increasing $\alpha$ and decreasing $\beta$, thus improving jet performance, without incurring in jet splitting. This result confirms and expands also previous studies which report a temperature dependence of jet performances, in particular transverse momentum spread, which is observed to decrease with jet cooling through nozzle cooling [24]. However, the lower limit on temperature requirement comes from the increased cluster formation efficiency at low temperatures, thus limiting the usable pressure ratios $R$ and hence jet density. The choice of $T$ will therefore depend on the density requirements of the jet and on the cooling systems available. Indeed, higher temperature jets remain more convenient and economical to use if large skimmer apertures are used, and use of room temperature jet has been successfully reported [25].

The simulations confirm the expected requirement of the skimmer depth SD to be as large as possible, preventing skimmed out molecules to affect the supersonic flow in front of the skimmer. SD is therefore only limited by geometrical consideration in the setup and manufacturing technology. In practice, for jets with high reservoir pressures up to $20 \mathrm{bar}$, SD should be chosen to be larger than $50 \mathrm{~mm}$.

Finally, the simulations show that geometric ratio is optimized for normalized nozzle-skimmer distance between 5 and 7 and skimmer width between 16 and 20. Larger skimmer widths sacrifice $G_{R}$ and $K$ for slightly better $H_{\rho}$.

Given the importance that gas speed and internal jet temperature has in many jet applications (e.g. molecular physics and spectroscopy), an analogous analysis as the one discussed above has also been performed focusing on these other observables. For this study, the reader is referred to a previously published work [26].

\section{Density profile}

To give a complete description of the jet-flow we consider the density profile shape of the created screen, whose features dramatically change depending especially on $T$ and $R$ in the perpendicular nozzle-skimmer slits configuration under analysis. Fig. 8 shows typical density profiles of the screen created for high pressure ratios $(R=5000)$ and large aperture angle $\alpha\left(25^{\circ}\right)$ and $\beta$ $\left(25^{\circ}\right)$, when lower $(150 \mathrm{~K})$ or higher $(300 \mathrm{~K})$ high pressure reservoir temperatures, respectively, are used. The density shown in the picture is calculated by integration of the gas-jet density across the 
a

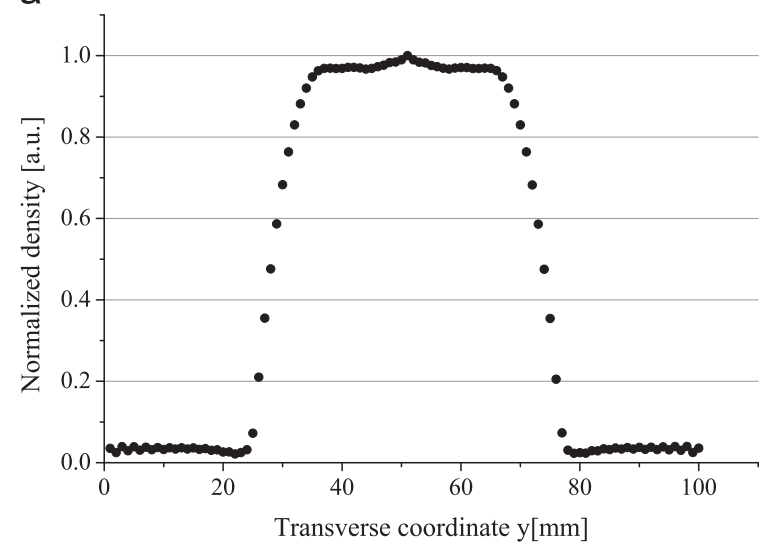

b

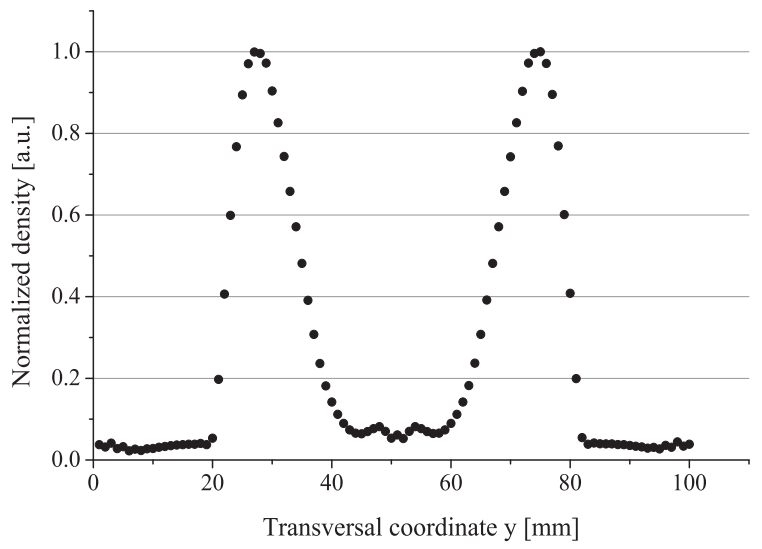

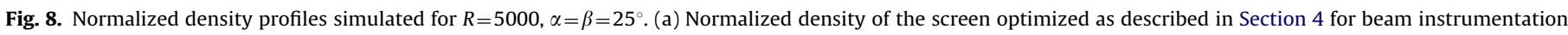

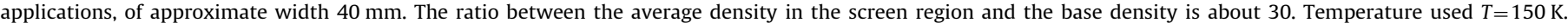

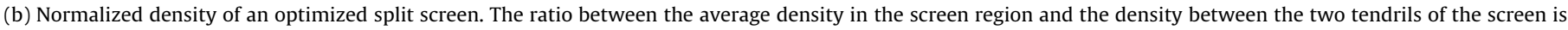

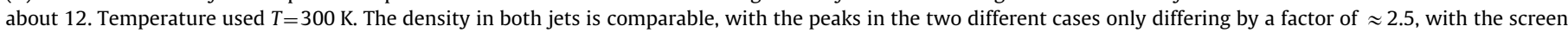
jet peak (a) being more dense than the split one (b).

screen short dimension, along coordinate $z$. The procedure is repeated for all values of the horizontal coordinate $x$ parallel with the jet expansion, and an average is taken and plotted for each $y$ value, with y being hence parallel to the screen long dimension. As there is nearly no variation of the screen density along the $\mathrm{x}$ coordinate in the range considered $(3 \mathrm{~cm})$, small with respect to the distance to the nozzle ( $>60 \mathrm{~cm}$ ), the average reported in Fig. 8 does not deviate from any single value by more than about $3.5 \%$. The simulations yielding the results in Fig. 8 feature a $G_{R}$ of about 40 , corresponding to a screen depth of about $1 \mathrm{~mm}$ for the $40 \mathrm{~mm}$ screen width shown in Fig. 8.

A screen splitting phenomenon is clearly seen in Fig. $8 \mathrm{~b}$, in which the screen density drops abruptly by more than one order of magnitude in the center, due to the development of tendril-like shock wave patterns known to form in the presence of shear layers typical of free jet expansion in the surroundings of the Mach disk. In fact, if the nozzle-skimmer parameters are conveniently adjusted, it is possible to have a secondary Mach disk created beyond the skimmer, leading to shear layers, tendril-like shock waves pattern and hence screen splitting. Our simulations show this phenomenon to occur for high temperatures together with high $G_{R}$.

Such phenomenon bears a potential for application in e.g. accelerator beam instrumentation, for beam halo monitoring or even soft beam scraping [27]. As it can be seen from Fig. 5a when operating in the regime of simulation \#242 it only takes a temperature variation to bring the system in the regime of simulation \#250, where screen splitting occurs. This shows another advantage of the proposed system, in the flexibility of changing operation mode by adjusting the thermodynamic properties of the gas reservoir, without having to modify any mechanical feature.

\section{Conclusions}

We have presented a study on the effect of geometry and thermodynamic variables (in the form of pressure ratio $R$ and temperature $T$ of the gas reservoir) on the generation of planar gas-jets. Focusing on applications like e.g. beam instrumentation, in which the major concern is the creation of a thin, homogeneous gas screen, we introduced a set of quality factors, which can be used for assessment of the planar jet, and used them to compare the effects of different nozzle-skimmer geometries, leading to the proposal of a novel, perpendicular nozzle-skimmer slits design, which outperforms traditional configurations by a factor of about 2 to 3 in homogeneity and geometric ratio and a factor of 2 in confinement. Furthermore, we presented a numerical study of the impact of the different individual geometric variables, calling upon the physics of the supersonic expansion shock wave pattern to justify our results. Finally, we highlighted the presence of a split screen phenomenon predicted to occur at higher stagnation temperatures when high $G_{R}$ are pursued, which can be exploited for application in e.g accelerator beam instrumentation.

\section{Acknowledgments}

The author acknowledges the useful discussions held with Dr. K.-U. Kuehnel and in particular with Dr. Y. Hashimoto on the technology and state of the art of planar gas-jet generation. The work presented in this paper is supported by the EU under contract PITN-GA-2008-215080, by the Helmholtz Association of National Research Centers (HGF) under contract number VH-NG328 and GSI Helmholtz Center for Heavy Ion Research GmbH.

\section{References}

[1] H.C. Man, J. Duan, T.M. Yue, Journal of Materials Processing Technology 63 (1997) 217

[2] A.V. Zaitsev, O.B. Kovalev, A.G. Malikov, A.M. Orishich, V.B. Shulyat'ev, Quantum Electronics 37 (2007) 891.

[3] R.E. Smalley, L. Wharton, D.H. Levy, Accounts of Chemical Research 10 (1977) 139.

[4] V. Rozhansky, I. Senichenkov, I. Veselova, D. Morozov, R. Schneider, Nuclear Fusion 46 (2006) 367.

[5] R. Dorner, J.M. Feagin, C.L. Cocke, H. Brauning, O. Jagutzki, M. Jung, E.P. Kanter, H. Khemliche, S. Kravis, V. Mergel, M.H. Prior, H. SchmidtBocking, L. Spielberger, J. Ullrich, M. Unversagt, T. Vogt, Physical Review Letters 77 (1996) 1024.

[6] V. Mergel, M. Achler, R. Dorner, K. Khayyat, T. Kambara, Y. Awaya, V. Zoran, B. Nystrom, L. Spielberger, J.H. McGuire, J. Feagin, Y. Azuma, H. SchmidtBocking, Physical Review Letters 80 (1998) 5301.

[7] J. Ullrich, R. Moshammer, A. Dorn, R. Dorner, L.P.H. Schmidt, H. SchmidtBocking, Reports on Progress in Physics 66 (2003) 1463.

[8] A. Amirav, U. Even, J. Jortner, Chemical Physics Letters 83 (1981) 1.

[9] R. Galiana, D. Manglunki, C. Mazeline, in: IEEE Particle Accelerator Conference, San Francisco (1991) p. 1198.

[10] M. Putignano, K.-U. Kuhnel, C.-D. Schroter, C.P. Welsch, Hyperfine Interactions 194 (2009) 189.

[11] C.P. Welsch, M. Grieser, J. Ullrich, A. Wolf, Nuclear Instruments and Methods in Physics Research Section A: Accelerators, Spectrometers, Detectors and Associated Equipment 546 (2005) 405. 
[12] M. Jugroot, C.P.T. Groth, B.A. Thomson, V. Baranov, B.A. Collings, Journal of Physics D: Applied Physics 37 (2004) 1289.

[13] S. Zhang, X. Ma, H. Liu, B. Li, X. Zhu, Science in China, Series G: Physics Mechanics and Astronomy 49 (2006) 709.

[14] K. Luria, W. Christen, U. Even, Journal of Physical Chemistry A 115 (2011) 7362.

[15] R. Subramanian, M. Sulkes, Review of Scientic Instruments 79 (2008) 016101.

[16] Y. Hashimoto, Y. Fujita, T. Morimoto, S. Muto, T. Fujisawa, T. Honma, K. Noda, Y. Sato, S. Yamada, in: Proceedings of the Second Asian Particle Accelerator Conference, Beijing, China, 2001, p. 791.

[17] Y. Hashimoto, Y. Fujita, S. Muto, T. Fujisawa, T. Furukawa, T. Homma, K. Noda, Y. Sato, H. Uchiyama, S. Yamada, A. Morinaga, J. Takano, K. Takano, in: Proceedings of EPAC, Paris, France, 2002, p. 1900.

[18] Y. Hashimoto, T. Fujisawa, T. Morimoto, Y. Fujita, T. Honma, S. Muto, K. Noda, Y. Sato, S. Yamada, Nuclear Instruments and Methods in Physics Research Section A: Accelerators, Spectrometers, Detectors and Associated Equipment 527 (2004) 289.
[19] T. Fujisawa, Y. Hashimoto, T. Morimoto, Y. Fujita, Nuclear Instruments and Methods in Physics Research Section A: Accelerators, Spectrometers, Detectors and Associated Equipment 506 (2003) 50.

[20] A.V. Zibarov, in: Proceedings of ASME, Pressure Vessel and Piping Conference 397-1 (1999) p. 117.

[21] H.R. Murphy, D.R. Miller, Journal of Physical Chemistry 88 (1984) 4474.

[22] D.R. Miller, in: Ed.,in: Giacinto Scoles (Ed.), Atomic and Molecular Beam Methods, vol. 1, Oxford University Press, NY, USA, 1988, p. 14.

[23] D. Givoli, Journal of Computational Physics 94 (1991) 1.

[24] I. Orlanski, Journal of Computational Physics 21 (1976) 251.

[25] O.F. Hagena, W. Obert, Journal of Chemical Physics 56 (1972) 1793.

[26] R. Dörner, V. Mergel, O. Jagutzki, L. Spielberger, J. Ullrich, R. Moshammer, H. Schmidt-Böcking, Physics Reports 330 (2000) 95.

[27] M. Putignano, C.P. Welsch, in: Proceedings of the Beam Instrumentation Workshop, Jacow, Santa Fe, USA 2010. 